\title{
Burning cups and donuts: what coherent X-rays can reveal about topological defects
}

\author{
D. Karpov ${ }^{1}$, J. Llandro², E. Fohtung ${ }^{3}$ \\ ${ }^{1}$ European Synchrotron Radiation Facility, 71 Avenue des Martyrs, 38000 Grenoble, France, ${ }^{2}$ Tohoku University, Laboratory for \\ Nanoelectronics and Spintronics, Sendai 980-8577, Japan, ${ }^{3}$ Department of Materials Science and Engineering, Rensselaer \\ Polytechnic Institute, NY 12180 Troy, USA
}

dmitry.karpov@esrf.fr

Topological defects are at the heart of many intriguing phenomena in fields as diverse as biology and materials science. Ability to manipulate the topological order at will has transformative implications for nanotechnology, particularly for next generation spintronic devices, solar cells, photonics, reconfigurable electronics, catalysts, and energy and information storage. To achieve such control, we must deepen our understanding of topological textures. It is therefore essential to comprehend their nature in 3D.

While electron microscopy methods achieve very high spatial resolution even in $3 \mathrm{D}$, for this they rely on destructive slicing/milling techniques that (1) induce excessive strain on the samples, potentially significantly altering the energy landscape; and (2) render timedependent studies impossible. On the other hand, X-rays have high penetration depth that allows them to access whole-volume information and are (mostly) non-destructive, preserving the structures under study. Moreover, X-rays do not interfere with electric and magnetic fields (as well as with visible light photons), allowing studies to be performed under external influences.

In this talk, we will show how Bragg coherent diffractive imaging, with help of Landau phase-field modelling, can be extended to the studies of ferroelectric domains, polar vortices [1] and 1D strings [2] in individual nanoparticles under external electric fields. Our results show that topological structures in ferroic materials can modulate the structural phase transition driven by electric field. When analyzing projections of toroidal moment, we also observed controllable chirality, which can be applied in next generation electronics. Tracking of the domain morphology and the vortex core lines suggests that some ferroic materials feature topological structures of the same universality class as hypothetical cosmic strings. This suggests that our methodology can be applied to the studies as exciting and fundamental as cosmology. We will further discuss how the same methodology can be adapted to the studies of large-scale topological textures in photonic networks imaged using ptychographic X-ray computed tomography [3]. We will emphasize the similarities between imaged topological entities and discuss implications of next generation synchrotron sources for the field.

a

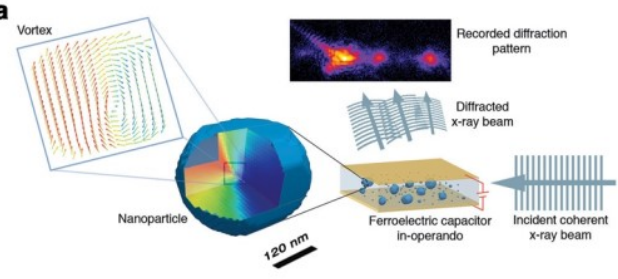

b

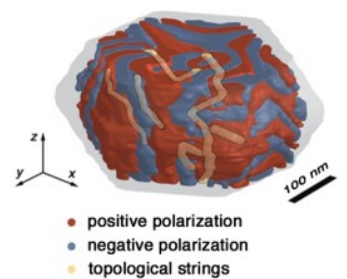

Figure 1. a, principle experimental scheme of Bragg coherent diffractive imaging experiment of a vortex in a $\mathrm{BaTiO}_{3}$ nanoparticle under external electric field [1]. b, rendering of ferroelectric 1D strings in individual nanoparticle of $\mathrm{BaFe}_{12} \mathrm{O}_{19}$ [2].

[1] D. Karpov, Z. Liu, T. dos Santos Rolo, R. Harder, P. V. Balachandran, D. Xue, T. Lookman, and E. Fohtung, "Three-dimensional imaging of vortex structure in a ferroelectric nanoparticle driven by an electric field", Nat. Comm. 8, 280 (2017)

[2] D. Karpov, Z. Liu, A. Kumar, B. Kiefer, R. Harder, T. Lookman, and E. Fohtung, "Nanoscale topological defects and improper ferroelectric domains in multiferroic barium hexaferrite nanocrystals", Phys. Rev. B 100, 054432 (2019)

[3] High-resolution three-dimensional imaging of topological textures in gyroid networks (manuscript in preparation).

Keywords: topological defects; Bragg coherent diffractive imaging; ptychographic X-ray computed tomography; ferroelectric materials; photonic structures 